\title{
Simulation and Implementation of Grid Tied Solar Photovoltaic System with Fuzzy Control Based Universal Bridge Inverter
}

\author{
Usha Verma \\ M.Tech Scholar \\ Scope College of Engineering \\ Bhopal, Madhya Pradesh, India \\ verma.usha6@gmail.com
}

\author{
N K Singh \\ Assistant Professor \\ Scope College of Engineering \\ Bhopal, Madhya Pradesh, India \\ dannyk0809@gmail.com)
}

\begin{abstract}
Worldwide renewable energy resources, especially solar energy, are growing dramatically in view of energy shortage and environmental concerns. Large-scale solar photovoltaic (PV) systems are typically connected to medium voltage distribution grids, where power converters are required to convert solar energy into electricity in such a grid-interactive PV system. This study are designing of solar energy system in MATLABSIMULINK environment which can be integrated with the grid for its efficient operation. The grid integration is necessary to ace the system reliable under various environmental conditions. Enhancing the DC input voltage to the inverter so that in its aspect the $\mathrm{AC}$ output from the inverter is also enhanced. And designing of a universal bridge inverter and AI based intelligent control for it such that it enhances the power output from the solar PV system. Designing of efficient rules for the inverter control using FUZZY algorithm. This work proposes an optimized active power enhancement method and evaluates the effect of fuzzy based controller for power enhancement on system reliability and power quality in the gridinteractive PV system with cascaded converter modules. Fuzzy set of rules are defined in a manner such that it is proved to be effective in enhancing the current output keeping the grid voltage same and hence the power output from the systems of cascaded PV modules. it can be concluded that if designing a cascaded PV solar system it is possible to increase the active power output from the inverter just by using fuzzy set of rules for firing pulses in the inverter.
\end{abstract}

Keywords: PV system, AC, DC, solar system.

\section{INTRODUCTION}

Global renewable energy sources, especially solar energy, are increasing dramatically in the face of energy shortages and environmental challenges. Large-scale photovoltaic systems (photovoltaic systems) are generally connected to medium voltage distribution networks where power converters are required to convert solar energy into electricity in such an interactive photovoltaic system. In order to gain direct access to the medium voltage network without the bulky medium voltage transformer, multilevel cascade converters are becoming more and more attractive due to their unique advantages such as improved power generation through monitoring. , with lower cost, higher power density, scalability and modularity of the maximum distributed power point (MPPT) and better energy efficiency, Plug-n-Power operation, etc. Although multistage cascade converters have been successfully introduced in medium and high voltage applications such as large motors, dynamic voltage restorations and reactive power compensation. Flexible AC transformers are used in photovoltaic systems due to the variability of solar energy and the mismatch of the maximum power point of individual conversion modules due to manufacturing tolerances, partial shading, dirt, thermal differences, etc. must meet network codes or requirements. The system adds up all the AC output voltage.

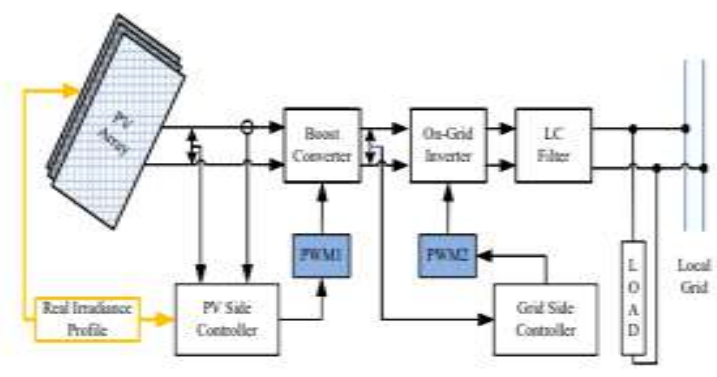

Fig. 1 Block diagram of the PV array power system

\section{LITERATURE REVIEW}

Liming Liu et.al [1] This paper discusses these issues, examines the effects of reactive power compensation and optimization on system reliability and quality, and proposes coordinated distribution of active and reactive power to address this problem. First, a vector method is developed to illustrate the principle of energy distribution. Therefore, the relationship between power and voltage is analyzed over a wide operating range. Therefore, an Optimized Reactive Power Compensation (RPCA) algorithm is proposed to 
improve the stability and reliability of system operation, facilitating the implementation of MPPT for each drive module. In addition, a complete control system with RPCA has been developed to achieve efficient power distribution and dynamic voltage regulation.

Mr. Benghanem et al. [2] General-purpose fossil fuel production addresses issues such as greenhouse gas emissions, environmental threats and energy crises. Let's build a single diode model with blocks from the MATLAB / Simulink library. Simulations were performed to determine the irradiation of $400 \mathrm{Wm} \& \mathrm{~min}$; from 2 to $1000 \mathrm{Wm}$ and above; ${ }^{2}$ vary. The simulation data is processed in Matlab. Consequently, the radiation accident on the module has a direct effect on the current delivered by the module, which in turn affects the fill factor and efficiency.

F. A. Salem [3] This overview provides various general and innovative mathematical models as well as Simulink models of photovoltaic (PV) systems based on the desired readability and specific application. The proposed models are developed and tested so that the designer has the maximum of visual and graphic digital data, selection, design and analysis of a particular photovoltaic system based on the desired performance and performance under certain conditions. Business. To get the desired output for specific systems. To meet the application requirements. This document also contains MATLAB scripts for calculating and recording the $\mathrm{I}-\mathrm{V}$ and $\mathrm{P}-\mathrm{V}$ properties of a particular PV system.

Ghazali et al. [4] The main objective of this study was to measure and determine the efficiency of polycrystalline, monocrystalline and amorphous silicon solar modules using a uniaxial solar tracker for the installation of photovoltaic modules (dynamic system). Module performance in a hot and humid climate in Malaysia. The dynamic system for this experiment was designed to support the three types of photovoltaic modules which allowed them to move from east to west (horizontally) with only one degree of freedom (horizontal) based on the orientation of the sun (from from alba) at sunset.

\section{OBJECTIVE}

The work is being focused on solar energy based system which is intended to meet following key objectives:

- Designing of solar energy system in MATLABSIMULINK environment which can be integrated with the grid for its efficient operation. The grid integration is necessary to ake the system reliable under various environmental conditions.

- Enhancing the DC input voltage to the inverter so that in its aspect the $\mathrm{AC}$ output from the inverter is also enhanced.
- Designing of a universal bridge inverter and AI based intelligent control for it such that it enhances the power output from the solar PV system.

- Designing of efficient rules for the inverter control using FUZZY algorithm. Fuzzy logic is applied with great success in various control application. A control system is an arrangement of physical components designed to alter another physical system so that this system exhibits certain desired characteristics.

- Enhancement of active power output from the solar energy system after using the proposed control algorithms.

\section{Methodology}

The grid connected photovoltaic systems are designed to operate parallel to the grid as shown. There are two general types of electrical designs for photovoltaic systems: systems that interact with the grid do not have a backup battery function and systems that interact and also include the backup battery. In the event of a fault, the device disconnects from the power supply and supplies certain load circuits. If the error occurs in daylight, the photovoltaic generator can take over the load to power the loads. The main component of both systems is the DC voltage inverter. The inverter is the key for the correct functioning of the system, but also for the most complex equipment. UPS requirements include operation on a wide range of voltages and currents, as well as regulated output voltage and frequency by providing alternating current.

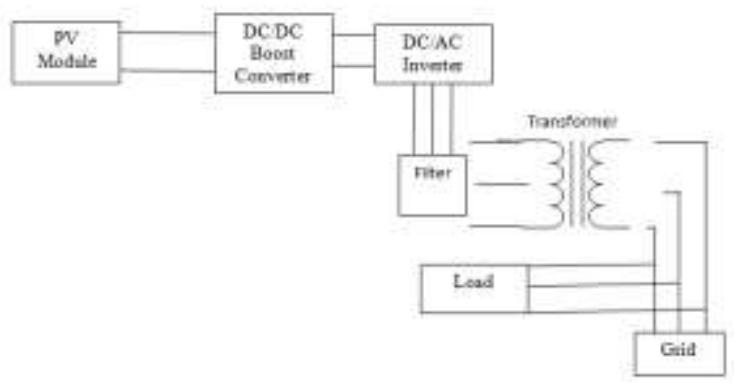

Fig. 2 Basic Block Diagram of solar system connected to the grid

PV Module modeling

PV cells have single operating point where the values of the current (I) and voltage (V) of the cell result in a maximum power output. These values correspond to a particular resistance, which is equal to V/I. A simple equivalent circuit of PV cell is shown in Fig. 3.3. 


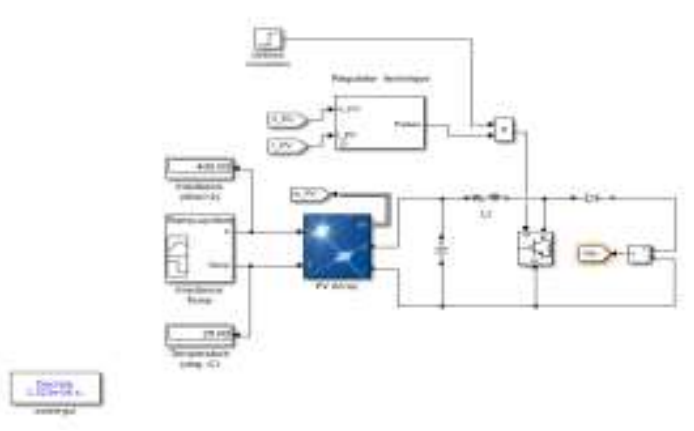

Fig. 3 Modeled solar system

A cell series resistance ( Rs ) is connected in series with parallel combination of cell photocurrent ( Iph), exponential diode (D), and shunt resistance (Rsh) , I pv and Vpv are the cells current and voltage respectively. It can be expressed as

$$
\begin{gathered}
I_{p v}=I_{p h}-I_{s}\left(e^{q\left(V_{p v}+I_{p v} * R_{s}\right) / n K T}-1\right)-\left(V_{p v}+I_{p v}\right. \\
\left.* R_{s}\right) / R_{s h}
\end{gathered}
$$

Where:

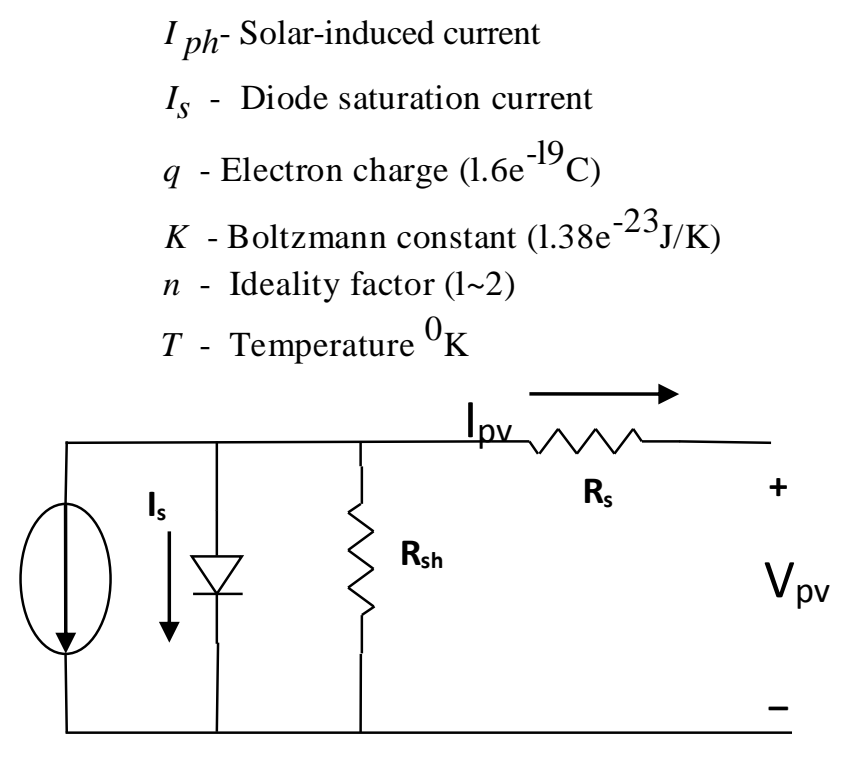

Fig. 4 Equivalent circuit of solar pv cell

The solar induced current of the solar PV cell depends on the solar irradiation level and the working temperature can be expressed as:

$$
I_{p h}=I_{s c}-k_{i}\left(T_{c}-T_{r}\right) * \frac{I_{r}}{1000}
$$

Where:

$I_{S C}$ Short-circuit current of cell at STC

$K_{i}$ Cell short-circuit current/temperature coefficient (A/K)

$I_{r}$ Irradiance in $\mathrm{w} / \mathrm{m}$

$T_{c}, T_{r}$ Cell working and reference temperature at STC

A PV cell has an exponential relationship between current and voltage and the maximum power point (MPP) occur at the knee of the curve as shown in the Fig 3.4.

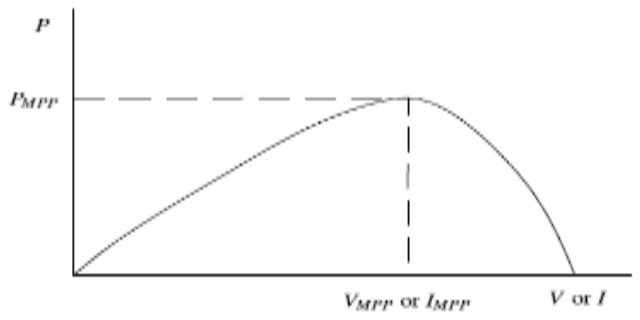

Fig. 5 Characteristic PV array power curve

The P\&O algorithm will track the maximum power to supply the DCMGs system. The assumptions for model derivation are that the ideal current source can be presented as the PVs behavior. In addition, all power converters are operated under the continuous conduction mode (CCM) and the harmonics are also ignored.

Grid system Modeling

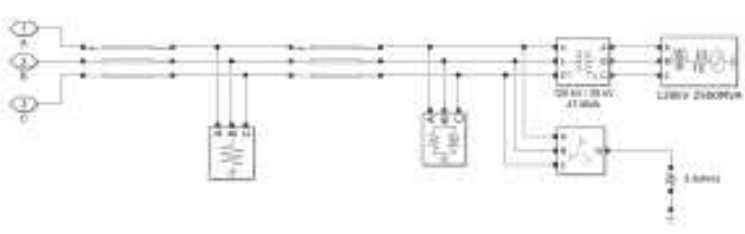

Fig. 6 MATLAB/SIMULINK model of grid system

\subsection{Universal Bridge Inverter}

The inverter system described in this document is a threephase VSI (Voltage Source Inverter) configuration that is commonly used in distributed generation interfaces. A PI synchronous frame current controller was selected to control the inverter.

If the electricity produced is transferred to the grid or used by AC loads, DC converters (inverters) must be used. The inverters can be single-phase or three-phase. There are four inverters integrated into the grid for photovoltaic systems: the central system inverter system, the string inverter system, the multi-strand inverter system and the micro-grid inverter system (AC modules).

The central inverter is the previous technology and is based on central inverters which have a large number of photovoltaic modules connected to the grid. The photovoltaic modules are connected in series (called strings). These strings are connected in parallel to the string diodes to achieve high power levels. String inverters are the current technology and represent the reduced type of central inverter that each string connects to the inverter. Multi-string inverters have multiple strings and are connected to a common DC-AC inverter with their own DC-DC converter. Thanks to their individual controllability, string inverters are better than central system 
inverters. The block diagram of the grid-connected threephase DC-AC inverter is shown in Figure 3.8.

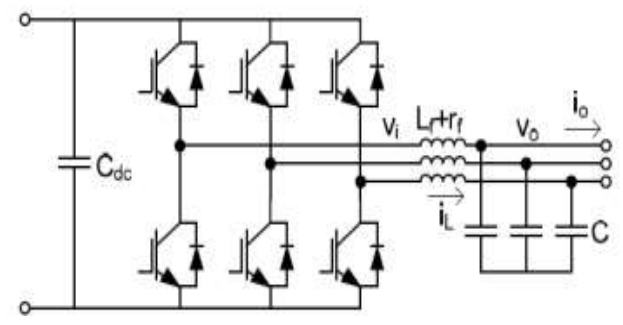

Fig. 7 Block diagram of three phase inverter.

\subsection{Fuzzy logic based inverter control}

Fuzzy logic is based on the concept of deciding output based on hypotheses. It works on a quantitative basis. Each sentence represents a language variable that defines the possible state of the output. Each possible entry state and degrees of state change are part of the package, depending on the expected exit. It basically works on the If-else-the principle, $\mathrm{i}$. H. If A AND B, then Z.

Suppose we want to control a system where the output can be anywhere in the set $X$ with a generic value $x$ such that $x$ belongs to $\mathrm{X}$. Consider a certain set $\mathrm{A}$, which is a subset of $X$, to which all members of $A$ they belong to the range 0 and 1. Set $A$ is known as fuzzy set and the value of $f A(x)$ in $x$ indicates the degree of membership of $x$ in this set. The output is decided based on the degree of belonging to $\mathrm{x}$ as a whole. This assignment of membership depends on the acceptance of the outputs according to the inputs and on the speed of variation of the inputs.

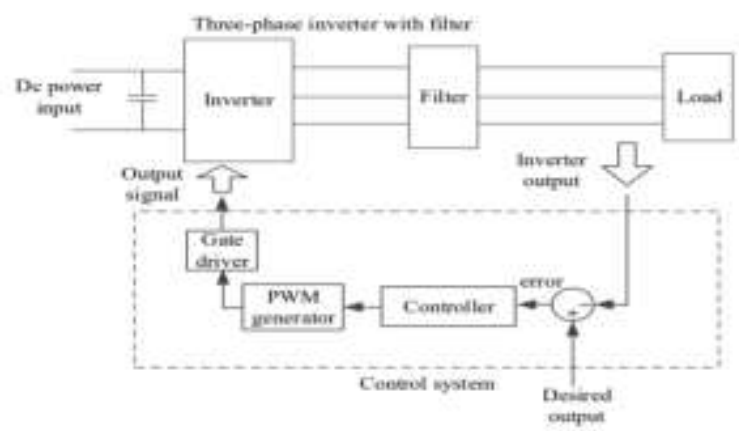

Fig. 8 Block diagram of proposed based inverter control

Each fuzzy logic block is made up of two inputs and one output. The first entry is the error and the other entry is the error rate, which is sampled once before the error values. The input and output membership functions are similarly named for all parameters of the transmission line. The conventional controller is replaced by a fuzzy controller. The input variables for the fuzzy controller are the error signal and the modification of this error.

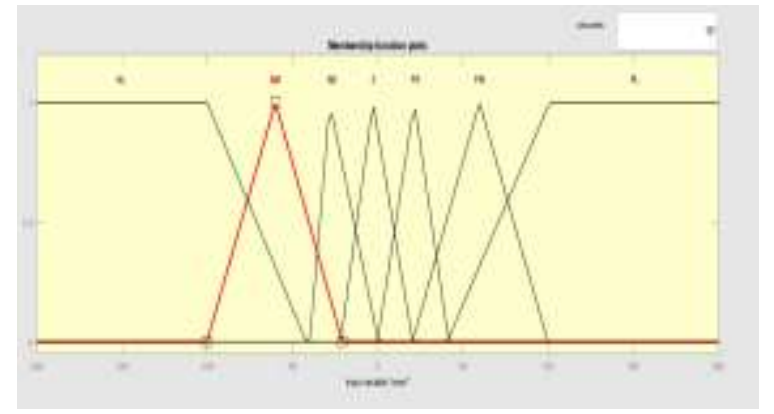

Fig. 9 Input Variable (error) Normalized Membership Function

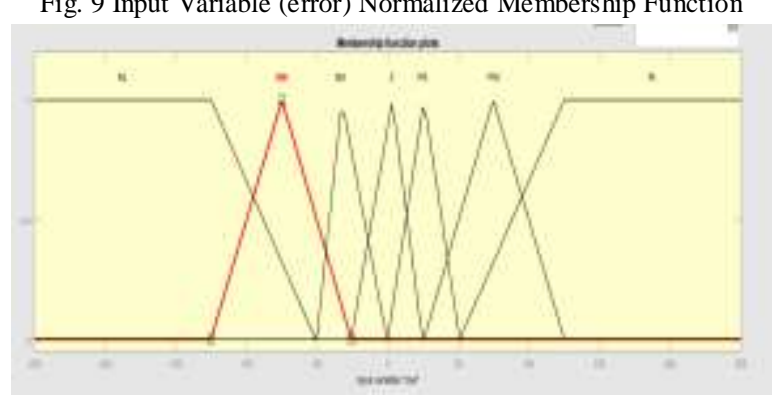

Fig. 10 Input Variable (coe) Normalized Membership Function

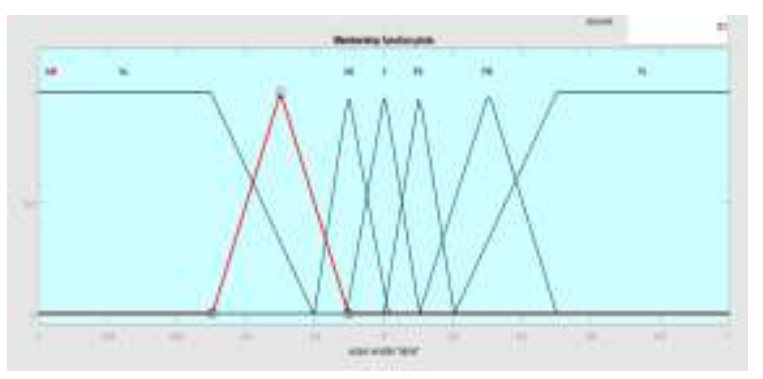

Fig. 11 Output Variable Normalized Membership Function Simulation is carried out for a fixed RES generation, which is greater than the load demand. The result of both scenarios is compared.

\section{RESULT}

Firstly the model is created in MATLAB/SIMULINK using power compensation with voltage source control, which is then integrated with the grid. The proposed work is being done by designing a inverter having control based on fuzzy logic inverter which is being modeled as to improve the current output from the system. The second model has been integrated with the grid alsoin order to enhance the efficiency and reliability of the system. This will ensure continuity in case the solar system is not working properly or is under maintenance. The model which is being proposed is expected to improve the active power output just by changing the inverter control system. This chapter discusses the modeling of the systems in following two cases:

CASE 1: Modeling the system with basic voltage source control for power enhancement 
CASE 2: Modeling the system with fuzzy logic based inverter control for active power enhancement

Solar irradiation is one of the important parameters that should be taken into consideration for the design and utilization of a photovoltaic system. Usually, the input parameters of a photovoltaic system are solar irradiation, the ambient environment temperature.

The outputs have been discussed in waveforms which has seen considerable imrovement in active power as well as Vdc input just by introducing a control in inverter. The temperature and irradiation being provided to the solar panels as a input is shown as below:

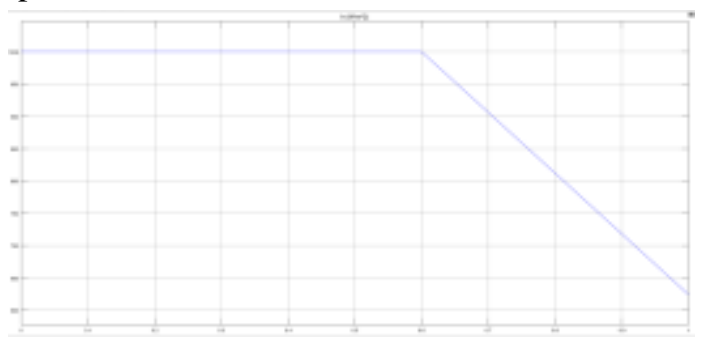

Fig. 12 Irradiation provided as an input to the solar panels $1000 \mathrm{wb} / \mathrm{m}^{2}$

The odel is being simulated to $1 \mathrm{sec}$ in MSIMULINK and the irradiation input to the solar panels is being varried for investigation.

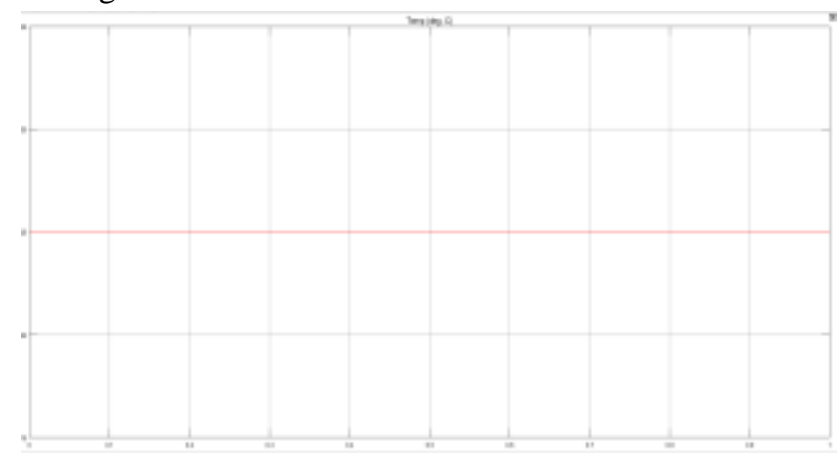

Fig. 13 Temperature provided as an input to the solar panels $250 \mathrm{C}$

The graph shows that the temperature of the solar module is being maintained to $25^{\circ} \mathrm{C}$. This temperature is maintained constant for the entire run time simulaiton.

CASE 1: Modeling the system with basic voltage source control for power enhancement

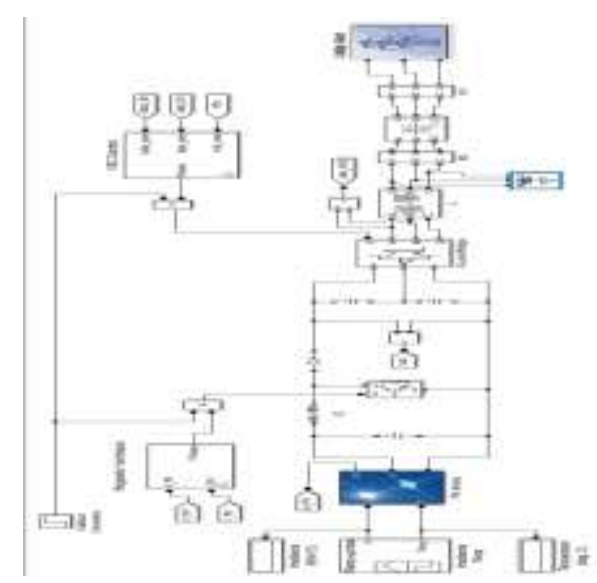

Fig. 14 MATLAB/SIMULINK model of solar system connected to grid with VSC control

Fig. 14 depicts the solar energy system which is being modeled with MATLAB/SIMULINK environment. The input to the solar cells is the variable radiation and temperature of $25^{\circ} \mathrm{C}$. The DC voltage which is obtained from the photovoltaic array of the solar panel has to be inverted. It can't be used to drive the load as it is of lower voltage and hence it has to be boosted to higher voltage. The DC output from the system is taken and fed to the boost converter with MPPT control algorithm driving it with the pulses. The DC output is then fed to the inverter for conversion from DC/AC power output. The AC output from the inverter is then fed to the grid via a transformer. The inverter is driven by a voltage source control.

The output voltage, current and active power output from the system is depicted in the graphs below.

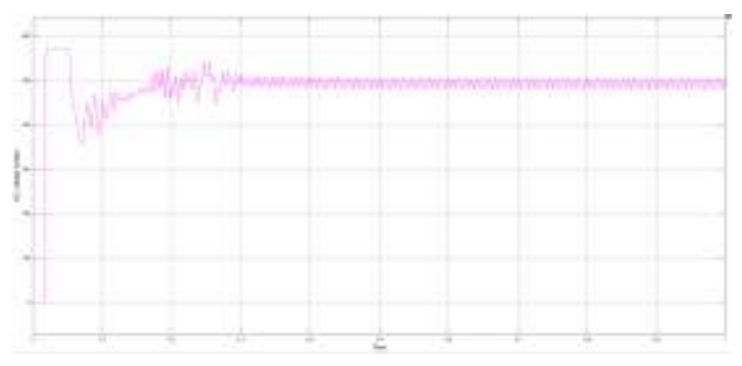

Fig. 15 DC input to the inverter in system having voltage source control

The fig. 15 shows the output DC voltage output from the solar PV system. The voltage output is read after boost converter. The value is approximately found to be 500 volts. This voltage is fed tot the inverter for further conversion. At this point basic DC operational devices can be connected for its operation. 


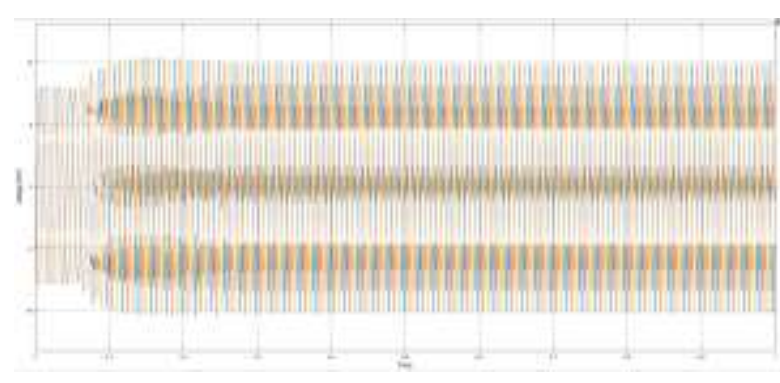

Fig. 16 Voltage output from the system

Fig. 16 Depicts voltage output from the system having inverter with basic voltage source control for generating pulses which are being fed to the inverter for its operation. The voltage output is $10 \mathrm{KW}$ AC which can be used for driving AC loads at 50 hertz frequency.

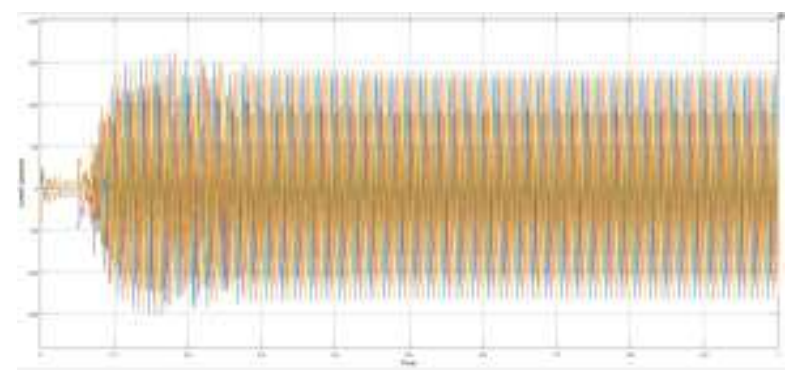

Fig. 17 Current output from the system

The fig 17 is showing the $\mathrm{AC}$ current output waveform from the solar energy system having basic voltage source controlled inverter. The figure shows that there is some amount of distortion present in the current output. The control is then further enhanced by changing the controlling algorithm for the inverter.

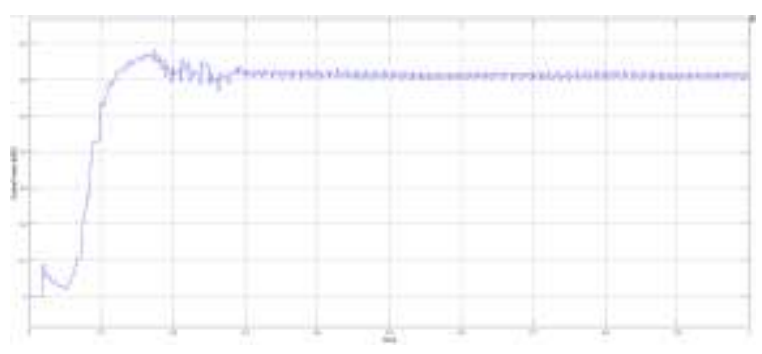

Fig. 18 Active Power output from the system

Fig. 18 shows the active power output from the system having inverter with basic voltage source controller. The active power output from the system is found to be approximately $0.62 \mathrm{MW}$. After this the PV solar panels or array are electrically connected or "tied" to the local mains electricity grid which feeds electrical energy back into the grid.

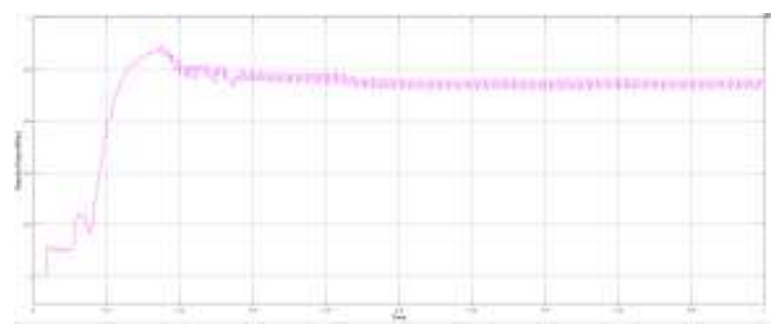

Fig. 19 Reactive Power output from the system.

A fig. 19 is showing the reactive power generated from the inverter control in Solar PV system which is also integrated with the grid. The Reactive power is the portion of electricity that helps establish and sustain the electric and magnetic fields required by alternating current equipment.

CASE 2: Modeling the system with fuzzy logic based inverter control for active power enhancement

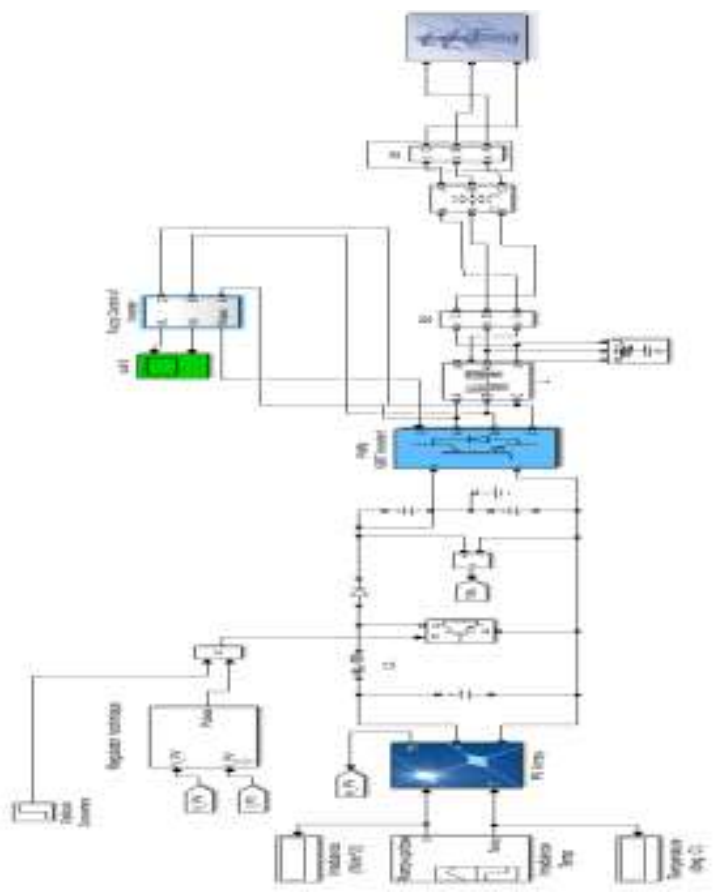

Fig. 20 MATLAB/SIMULINK model of solar system connected to grid with fuzzy logic based controller

The proposed solar power circuit consists of a solar generator, a step-up converter and a step-up converter. The power of solar energy that is supplied by the step-up inverter supply to the autonomous load without an intermediate conversion stage or filter. For the operation of the step-up converter, the duty cycle is modified through the fuzzy logic controller and the PWM block in order to adjust the converter output voltage.

The output voltage, current and active power output from the system is depicted in the graphs below. 


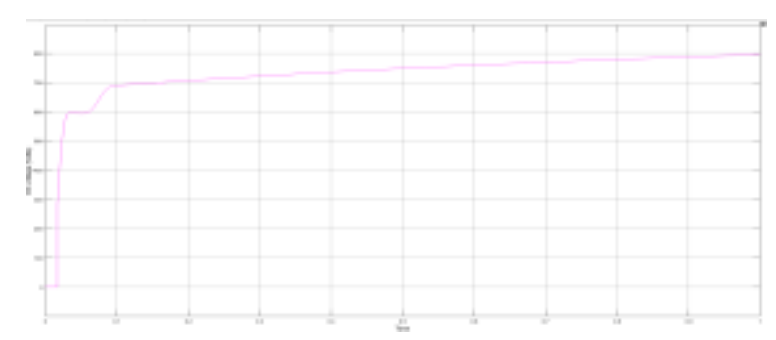

Fig. 21 DC input being fed to the inverter in system having fuzzy logic based controller

The figure 4.10 shows the output DC voltage output from the solar PV system in which the inverter is provided with the pulses driven from fuzzy based voltage control. The voltage output is read after boost converter. The value is approximately found to be 690 volts. The voltage profile found is being converter to AC and the power output is then fed to the grid via transformer.

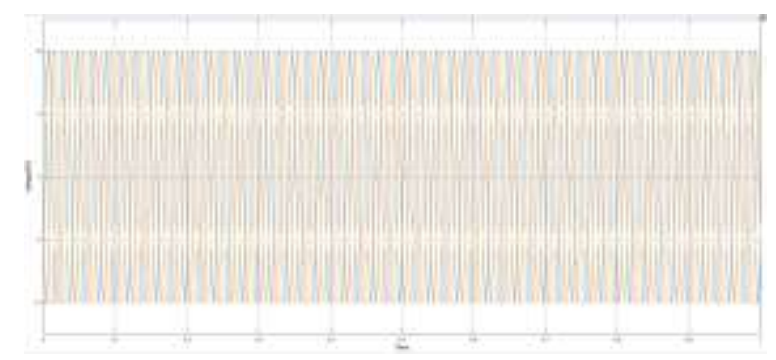

Fig. 22 Voltage output from the system having fuzzy logic controller

Fig. 22 Depicts voltage output from the system having inverter with fuzzy logic based voltage control for generating pulses which are being fed to the inverter for its operation. The voltage output is $10 \mathrm{KW}$ AC which can be used for driving AC loads at 50 hertz frequency. This voltage is kept same while improving the power profile of the system.

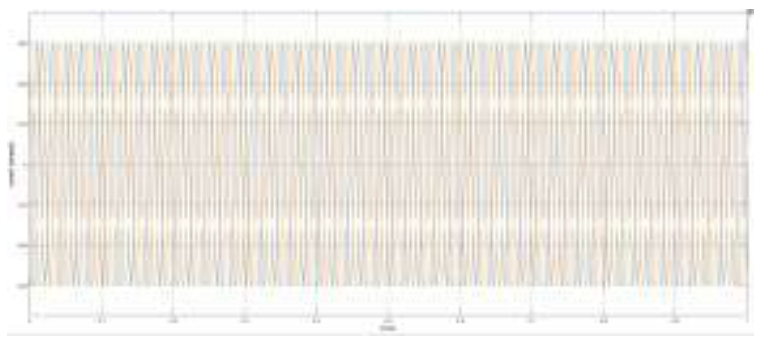

Fig. 23 Current outputs from the system having fuzzy logic controller

The fig. 23 is showing the AC current output waveform from the solar energy system having inverter controlled by designing of fuzzy logics for the gate pulses. The figure shows that distortion present in the current output is slightly reduced with this technique. This would result in the better active power outcome from the inverter as well.

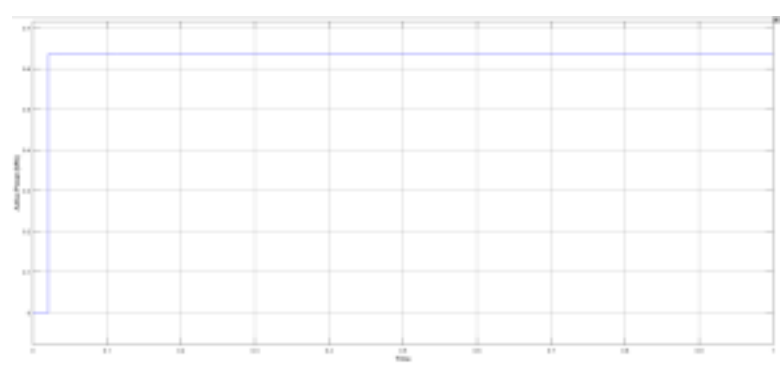

Fig. 24 Active Power output from the system having fuzzy logic controller

FLC requires the expert knowledge of the process operation for the FLC parameter setting, and the controller can be only as good as the expertise involved in the design. Fuzzy logic has the advantages to face the imprecise and uncertainty. The figure 5.13 is showing the active power output from the system in which inverter is being driven by a fuzzy logic based controller for generating pulses to be fed to it.

The active power output was found to be mores smooth and improved aw compared to the previous results. The power output is found to be approximately $0.63 \mathrm{MW}$ which is more stable and efficient in terms of feeding it to the load or grid.

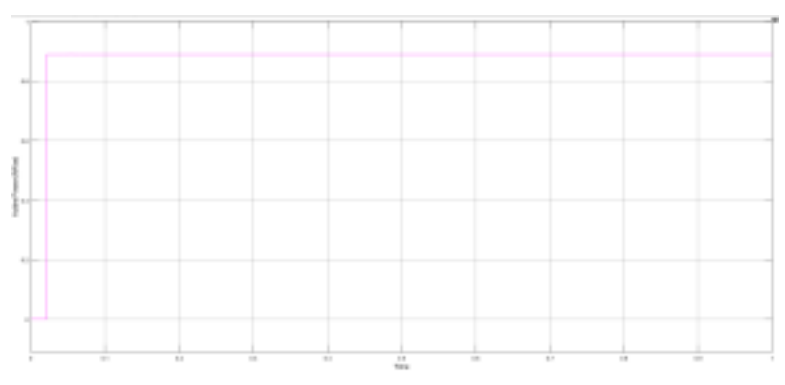

Fig. 25 Reactive Power output from the system having fuzzy logic controller

A fig. 25 is showing the reactive power generated from the inverter control in Solar PV system which is also integrated with the grid. The power can be utilized for driving loads which require compensation.

\subsection{Validation}

This chapter compares the usage of fuzzy logic based controller with that of the system without it. The cascaded solar PV system is being modeled in MATLAB/SIMULINK and is simulated for 0.5 seconds for observing the results. It is observed that the system sees an enhancement in the current and thereby the active power output keeping the voltage output same. 


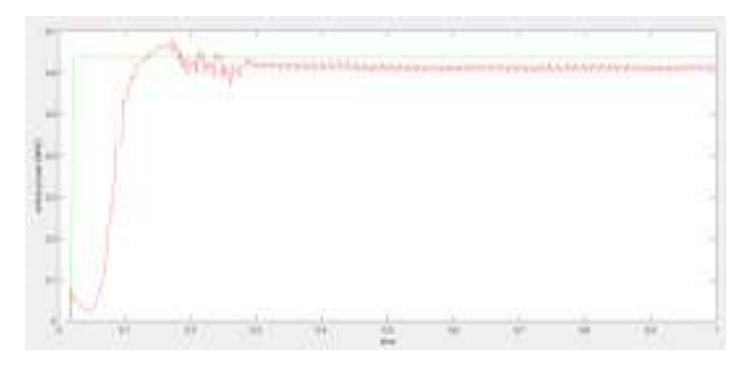

Fig. 26 Comparative Analysis of active power outputs

The system enhances the active power output and hence making it viable using the fuzzy based controller we can enhance the system rating.

\section{CONCLUSION}

Cascaded PV system configuration is presented. Basic I\&C technique is used to trace the maximum point voltage form the cascade systems.

This work proposes an optimized active power enhancement method and evaluates the effect of fuzzy based controller for power enhancement on system reliability and power quality in the grid-interactive PV system with cascaded converter modules. Fuzzy set of rules are defined in a manner such that it is proved to be effective in enhancing the current output keeping the grid voltage same and hence the power output from the systems of cascaded PV modules. The conclusion can be formulated below in the table as follows:

\begin{tabular}{|c|c|c|}
\hline \multicolumn{2}{|c|}{$\begin{array}{c}\text { Table 1: Comparison of outputs from the system having voltage source } \\
\text { control and cascaded system having inverter control using fuzzy logic } \\
\text { controller }\end{array}$} \\
\hline Parameters & $\begin{array}{c}\text { System with } \\
\text { voltage source } \\
\text { control without } \\
\text { fuzzy () }\end{array}$ & $\begin{array}{c}\text { Cascaded system with } \\
\text { fuzzy based control for } \\
\text { power enhancement }\end{array}$ \\
\hline Active power (MW) & 0.62 & 0.63 \\
\hline DC Voltage (volts) & 500 & 800 \\
\hline
\end{tabular}

The conclusion can be drawn in the following points:

- The proposed designing of the fuzzy rules for enhancing the active power output has been achieved in the cascaded system. The instantaneous active power output from the system without fuzzy control is $0.62 \mathrm{MW}$ and from the system having fuzzy controller the output is $0.6375 \mathrm{MW}$.

- Also the DC voltage is being improved from the 500 volts to 800 volts. This results in better input parameter to the inverter as compared to the case of system having no fuzzy controller

- The proposed set of fuzzy rules is easy to implement and also enhances the system efficiency by making it viable to be used for heavy loads with the same voltage. The proposed system is also then integrated with the grid to make it more and more reliable.

Hence it can be concluded that if designing a cascaded PV solar system it is possible to increase the active power output from the inverter just by using fuzzy set of rules for firing pulses in the inverter. The rules are so designed such that it increases the current output keeping the voltage output same and hence the active power output from the system. It can be concluded that the system efficiency is enhanced by using artificial intelligence based technique and hence can be implemented in any solar system.

The system is highly beneficial for distributed generation (DGs) in consumption of power in the local area as it is improving the active power output.

\section{Future Scope}

The proposed approach was demonstrated which can be extended, to be able to effectively enhance system operation stability and reliability, and improve power quality further. The modeled control of the inverter can be used in wind energy hybrid systems which are connected to the grid.

\section{REFERENCES}

[1] Liming Liu, Hui Li, YaosuoXue, Wenxin Liu," Reactive Power Compensation and Optimization Strategy for Grid-Interactive Cascaded Photovoltaic Systems" IEEE, Transactions on Power Electronics, DOI 10.1109/TPEL.2014.2333004.

[2] M. S. Benghanem, and S. N. Alamri, 2009, "Modeling of photovoltaic moduleand experimental determination of serial resistance," in Journal of Taibah University for Science JTUSCI, Vol. 2, pp. 94-105.

[3] F. A. Salem, 2014, "Modeling and Simulation issues on Photovoltaic systems, for Mechatronics design of solar electric applications," IPASJ International Journal of Mechanical Engineering IIJME, Vol. 2, Issue 8, pp. 24-47.

[4] M. Ghazali, and A. Rahman, 2012, "The Performance of Three Different Solar Panels for Solar Electricity Applying Solar Tracking Device under the Malaysian Climate Condition," in Canadian Center of Science and Education Energy and Environment Research, Vol. 2, No. 1, pp. 235-24

[5] J. Bauer, "Single Phase Voltage Source Inverter Photovoltaic Application", Actaolytechnica Vol. 50 No. 4, 2010.

[6] X. Renzhong, Xi. Lie, Z. Junjun, and D. Jie, "Design and Research onthe LCL Filter in Three-Phase PV Grid-Connected Inverters", International Journal of Computer and Electrical Engineering, Vol. 5,No. 3, June 2013.

[7] A. Arjun, B. Vind, N. Kumaresan and D.R.Binu Ben Jose, "A power Electronic Controller for PV-tied Grid-Connected system with single parameter sensing for MPPT using Boost Converter and LineCommutated Inverter", IEEE ICSET, Nepal, 2012.

[8] J. Li, F. Zhuo, X. Wang, L. Wang and S. Ni, "A Grid-Connected PVSystem with Power Quality Improvement Based on Boost + DualLevelFour-Leg Inverter", IEEE IPEMC, 2009. 